\title{
Flow characteristics analysis for flow past the porous spheres: wake structures
}

\author{
Likun $\mathrm{Ma}^{13}$, Sina Kashanj ${ }^{2}$, Shuliang Xu${ }^{1}$, Mao Ye ${ }^{1}$, David S Nobes ${ }^{2 *}$ \\ ${ }^{1}$ Dalian Institute of Chemical Physics, Chinese Academy of Sciences, Dalian, P.R.China \\ ${ }^{2}$ University of Alberta, Department of Mechanical Engineering, Edmonton, Canada \\ ${ }^{3}$ University of Chinese Academy of Sciences, Beijing, P.R.China \\ *dnobes@ualberta.ca
}

\begin{abstract}
Flow past a permeable sphere is different from that of a solid sphere due to the penetration of the fluid within porous structures, which can arise a change of flow fields. In this work, flow past porous spheres with Darcy numbers $(D a)$ ranging from $\left[0,10^{-3}\right]$ were measured using planar Particle Image Velocimetry (PIV). The whole flow fields, including both leading edge and trailing edge, were captured at six different Reynolds numbers $(R e)$ varying from 400 to 1400 . Time-average flow fields were calculated based on instantaneous flow fields within fully-developed stages. Local minimum method was used to search for stagnation point positions. The results show positions of stagnation points are nearly proportional to the logarithm of $R e$. For most porous spheres, positions of stagnation points are extended to farther downstream positions than that of a solid sphere. However, at some certain Darcy numbers, ranging from $5 * 10^{-6}$ to $2 * 10^{-5}$, positions of stagnation points are closer to the sphere centers than that of an impermeable one.
\end{abstract}

\section{Introduction}

The flow through and around a group of particles or a permeable body is widely seen in many industrial processes, including the motion of clusters in fluidized bed reactors, movement of coal particles in power stations and transport of porous microcarriers in bioreactors. Understanding flow characteristics, especially wake structures and fluid-particle interaction forces, of permeable bodies which are different from solid bodies, is important for the control of these processes Yu et al. (2012).

Theoretical analysis of flow characteristics for flow past a porous sphere has been limited to the creeping flow regime where inertial effects can be neglected. For higher Reynolds numbers, many numerical simulations studies Wu and Lee (1999); Wittig et al. (2017) have been carried out and it has been found Yu et al. (2012) the length of wake for flow past a porous sphere is different from that of a solid sphere and varies with permeability. A detached or penetrating wake is also observed for a certain permeability range, which is different from the attached wake of a solid sphere. Additionally, the changed wake structures can affect the force acting on the sphere, such as the pressure drag force. However, it's difficult for numerical simulations to determine appropriate fluid-porous interface boundary conditions for porous spheres, which can significantly affect the simulation results. Thus, there is a need for detailed experimental results of porous spheres as a benchmark to understand wake characteristics and validate numerical simulations results.

PIV is a non-intrusive measurement technique which can provide velocity field of full field with excellent temporal and spatial resolution. In this paper, the effect of the permeability for flow fields is investigated by applying PIV technique to visualize the difference of the flow past a solid sphere and a porous one.

\section{Experimental setup}

In our experiments, all spheres are connected to a traverse moving in horizontal direction which can be controlled with a controller to be given a constant velocity with $400 \leq R e \leq 1400$. 2D-PIV is used to 
capture time-dependent flow field and a double frame CCD camera is synchronized to a double pulse laser to capture images of fluid flow seeded with $18 \mu \mathrm{m}$ hollow glass particles. Both camera and the sphere are connected to the traverse to capture the images in an Eulerian reference frame.

\section{Results and discussion}

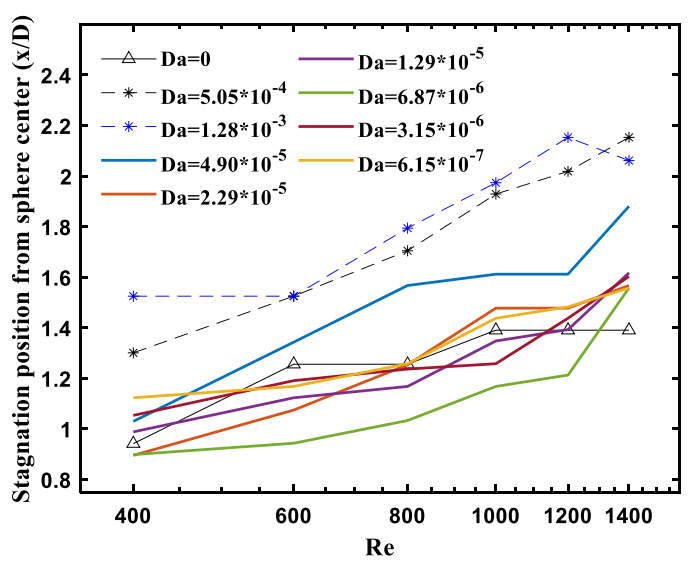

Figure 1: Variation of stagnation points positions along $R e$ at nine different Darcy numbers

Darcy numbers is dimensionless permeability, which can be written as $D a=k / D^{2}=d^{2} \cdot \varepsilon^{3} /\left(D^{2} \cdot 180\left(1-\varepsilon^{2}\right)\right)$. In this study, flow fields of porous spheres with Darcy numbers ranging from $\left[0,10^{-3}\right]$ were captured at $R e=400,600,800,1000,1200,1400$. Instantaneous frames in fully-developed stage were used to calculate time-average flow fields. Local minimums of the vector field were regarded as stagnation point positions. It can be found for a high permeability when $D a$ is more than $5.05 * 10^{-4}$, as shown in Figure 1 positions of stagnations points from sphere centers are significantly increased compared with that of a solid sphere. For most porous spheres, positions of stagnation points are extended to farther downstream positions than that of a solid sphere while it is only increased slightly for the low permeability spheres. However, at some certain Darcy numbers, ranging from $5 * 10^{-6}$ to $2 * 10^{-5}$, positions of stagnation points are closer to the sphere centers than that of an impermeable one. These phenomena are because, for a large permeability, a higher percentage of fluid will go through rather than flow around the porous sphere, causing a decrease on the effective $R e$ with a closer position of stagnation point. The penetrating flow also pushes the stagnation point's position away from the porous sphere, causing farther stagnation points. Thus, the final position of stagnation points are depended on the combination of the above two opposite effects. It is also clear that positions of stagnation points are nearly proportional to the logarithm of $R e$ for all different permeability.

\section{Acknowledgements}

This work was finished in University of Alberta, Canada, supported from a scholarship by the University of Chinese Academy of Sciences, China.

\section{References}

Wittig K, Nikrityuk P, and Richter A (2017) Drag coefficient and nusselt number for porous particles under laminar flow conditions. International Journal of Heat and Mass Transfer 112:1005-1016

Wu R and Lee D (1999) Highly porous sphere moving through centerline of circular tube filled with newtonian fluid. Chemical Engineering Science 54:5717-5722

Yu P, Zeng Y, Lee T, Chen X, and Low H (2012) Numerical simulation on steady flow around and through a porous sphere. International Journal of Heat and Fluid Flow 36:142-152 